\title{
Diagnostic value of cardiovascular magnetic resonance in comparison to endomyocardial biopsy in cardiac amyloidosis: a multi-centre study
}

\author{
Grigorios Chatzantonis $^{1} \cdot$ Michael Bietenbeck $^{1} \cdot$ Ahmed Elsanhoury $^{2} \cdot$ Carsten Tschöpe $^{2,3} \cdot$ Burkert Pieske $^{4}$. \\ Gloria Tauscher $^{5}$. Julia Vietheer ${ }^{6,7} \cdot$ Zornitsa Shomanova $^{1} \cdot$ Heiko Mahrholdt $^{5}$. Andreas Rolf ${ }^{6,7} \cdot$ Sebastian Kelle ${ }^{4}$. \\ Ali Yilmaz ${ }^{1}(\mathbb{0})$
}

Received: 28 August 2020 / Accepted: 26 October 2020/ Published online: 10 November 2020

(C) The Author(s) 2020

\begin{abstract}
Background Cardiac amyloidosis (CA) is an infiltrative disease characterised by accumulation of amyloid deposits in the extracellular space of the myocardium-comprising transthyretin (ATTR) and light chain (AL) amyloidosis as the most frequent subtypes. Histopathological proof of amyloid deposits by endomyocardial biopsy (EMB) is the gold standard for diagnosis of CA. Cardiovascular magnetic resonance (CMR) allows non-invasive workup of suspected CA. We conducted a multi-centre study to assess the diagnostic value of CMR in comparison to EMB for the diagnosis of CA.

Methods We studied $N=160$ patients characterised by symptoms of heart failure and presence of left ventricular (LV) hypertrophy of unknown origin who presented to specialised cardiomyopathy centres in Germany and underwent further diagnostic workup by both CMR and EMB. If CA was diagnosed, additional subtyping based on EMB specimens and monoclonal protein studies in serum was performed. The CMR protocol comprised cine- and late-gadolinium-enhancement (LGE)-imaging as well as native and post-contrast T1-mapping (in a subgroup)—allowing to measure extracellular volume fraction (ECV) of the myocardium.

Results An EMB-based diagnosis of CA was made in $N=120$ patients (CA group) whereas $N=40$ patients demonstrated other diagnoses (CONTROL group). In the CA group, $N=114$ (95\%) patients showed a characteristic pattern of LGE indicative of CA. In the CONTROL group, only 1/40 (2\%) patient showed a "false-positive" LGE pattern suggestive of CA. In the CA group, there was no patient with elevated T1-/ECV-values without a characteristic pattern of LGE indicative of CA. LGE-CMR showed a sensitivity of $95 \%$ and a specificity of $98 \%$ for the diagnosis of CA. The combination of a characteristic LGE pattern indicating CA with unremarkable monoclonal protein studies resulted in the diagnosis of ATTR-CA (confirmed by EMB) with a specificity of $98 \%$ [95\%-confidence interval (CI) $92-100 \%$ ] and a positive predictive value (PPV) of $99 \%$ (95\%-CI 92-100\%), respectively. The EMB-associated risk of complications was $3.13 \%$ in this study-without any detrimental or persistent complications.

Conclusion Non-invasive CMR shows an excellent diagnostic accuracy and yield regarding CA. When combined with monoclonal protein studies, CMR can differentiate ATTR from AL with high accuracy and predictive value. However, invasive EMB remains a safe invasive gold-standard and allows to differentiate CA from other cardiomyopathies that can also cause LV hypertrophy.
\end{abstract}

Keywords CMR $\cdot$ CA $\cdot$ EMB $\cdot$ Scintigraphy $\cdot$ Immunofixation

\section{Electronic supplementary material The online version of this article (https://doi.org/10.1007/s00392-020-01771-1) contains supplementary material, which is available to authorized users.}

G. Chatzantonis and M. Bietenbeck contributed equally to this work.

Ali Yilmaz

ali.yilmaz@ukmuenster.de

Extended author information available on the last page of the article

\author{
Abbreviations \\ AL Immunoglobulin light chain amyloidosis \\ ATTR Transthyretin amyloidosis \\ CA Cardiac amyloidosis \\ CAD Coronary artery disease \\ CIED Cardiovascular implantable electronic device \\ CKD Chronic kidney disease \\ CMR Cardiovascular magnetic resonance \\ ECG Electrocardiogram
}




$\begin{array}{ll}\text { ECV } & \text { Extracellular volume } \\ \text { EMB } & \text { Endomyocardial biopsy } \\ \text { HCM } & \text { Hypertrophic cardiomyopathy } \\ \text { HFpEF } & \text { Heart failure with preserved ejection fraction } \\ \text { IQR } & \text { Interquartile range } \\ \text { LGE } & \text { Late-gadolinium enhancement } \\ \text { LV } & \text { Left ventricle } \\ \text { LV-EDV } & \text { Left ventricular end-diastolic volume } \\ \text { LV-EF } & \text { Left ventricular ejection fraction } \\ \text { LVH } & \text { Left ventricular hypertrophy } \\ \text { MGUS } & \text { Monoclonal gammopathy of undetermined } \\ & \text { significance } \\ \text { NPV } & \text { Negative predictive value } \\ \text { NSF } & \text { Nephrogenic systemic fibrosis } \\ \text { PPV } & \text { Positive predictive value } \\ \text { QALE } & \text { Query amyloid late enhancement } \\ \text { ROC } & \text { Receiver operating characteristic curve } \\ \text { ROI } & \text { Region of interest } \\ \text { sFLC } & \text { Serum-free light chain assay } \\ \text { sIFE } & \text { Immunofixation electrophoresis in serum } \\ \text { SPE } & \text { Serum protein electrophoresis } \\ \text { TTE } & \text { Transthoracic echocardiogram }\end{array}$

\section{Introduction}

Cardiac amyloidosis (CA) is caused by deposition of misfolded amyloid fibrils in the extracellular space of the myocardium resulting in a specific cardiomyopathy $[1,2]$. The two major forms of CA are transthyretin-related amyloidosis (ATTR) and immunoglobulin light-chain amyloidosis (AL), accounting for nearly $95 \%$ of cases [3]. ATTR comprises two subtypes: the acquired wild-type (wt) ATTR (wtATTR) and the hereditary or mutant ATTR (mATTR) that is caused by genetic mutations in the transthyretin (TTR) gene $[1,4]$.

Cardiovascular magnetic resonance (CMR) has emerged as a robust non-invasive imaging modality that offers comprehensive and detailed cardiac information regarding functional and structural data [5]. In particular, late-gadoliniumenhancement (LGE)-imaging allows to robustly detect a characteristic pattern of myocardial damage indicative of CA [6]. Moreover, novel CMR techniques such as T1-mapping and measurement of the extracellular volume fraction (ECV) of the myocardium promise an improved assessment of even subtle structural changes in the myocardium. Some recent single-centre studies suggested a higher diagnostic yield and prognostic value of CMR based on T1-mapping and ECV measurement compared to conventional LGE-imaging [7, 8]. However, multi-centre CMR data regarding the diagnostic yield of CMR parameters for the diagnosis of CA are still limited.

Despite considerable progress in non-invasive imaging modalities, invasive endomyocardial biopsy (EMB) is still considered the gold standard for workup of cardiomyopathies of unknown origin [9-11]. The need for invasive EMB was recently questioned by Gillmore et al. in case of suspected CA since the combined finding of a "positive" bone scintigraphy indicative of CA and the absence of monoclonal proteins resulted in a specificity as well as positive predictive value (PPV) of $100 \%$ for the diagnosis of cardiac ATTR [12].

In the present multi-centre study, we sought to assess the diagnostic value of CMR regarding the diagnosis of CA in patients with unexplained left ventricular hypertrophy (LVH) - in comparison to the current gold-standard EMB. Similar to previous scintigraphic studies [12], we analysed the specificity and PPV of CMR in patients with the combined finding of a "positive" CMR study indicative of CA and "negative" monoclonal protein studies.

\section{Methods}

\section{Study population}

The present study group comprised $N=160$ patients suffering from symptoms of heart failure in the presence of left ventricular (LV) hypertrophy (defined as maximal LV wall thickness of $\geq 12 \mathrm{~mm}$ ) that could not be explained by abnormal loading conditions and who were referred to four German specialised cardiomyopathy centres between 2016 and 2019 for further diagnostic workup. Only patients who underwent both non-invasive CMR and invasive EMB, respectively, were included in this retrospective study. Noteworthy, patients with e.g. typical CMR findings of hypertensive heart disease or hypertrophic cardiomyopathy (HCM) who did not routinely undergo EMB were not included in this analysis. Moreover, the participating German centres share similar diagnostic algorithms including EMB workup in every patient with suspected CA. If EMB workup resulted in the diagnosis of $\mathrm{CA}$, additional subtyping based on EMBspecimens was performed to differentiate ATTR from AL (and other forms). Furthermore, if CA was diagnosed based on CMR and/or EMB, additional monoclonal protein studies-comprising serum protein electrophoresis ( $\mathrm{SPE}$ ), serum immunofixation electrophoresis (sIFE) and serum-free light chain assay (sFLC) - were performed. In case of an EMBbased diagnosis of ATTR, additional genetic testing of the transthyretin gene was ordered to differentiate between wild-type (wt)-ATTR and mutant (m)-ATTR. Patients with an EMB-based diagnosis of CA (either ATTR or AL) were assigned to the CA group whereas patients with other cardiac diagnoses formed the CONTROL group (Fig. 1). Ethics approval was obtained by local authorities and written informed consent was obtained by participants. 


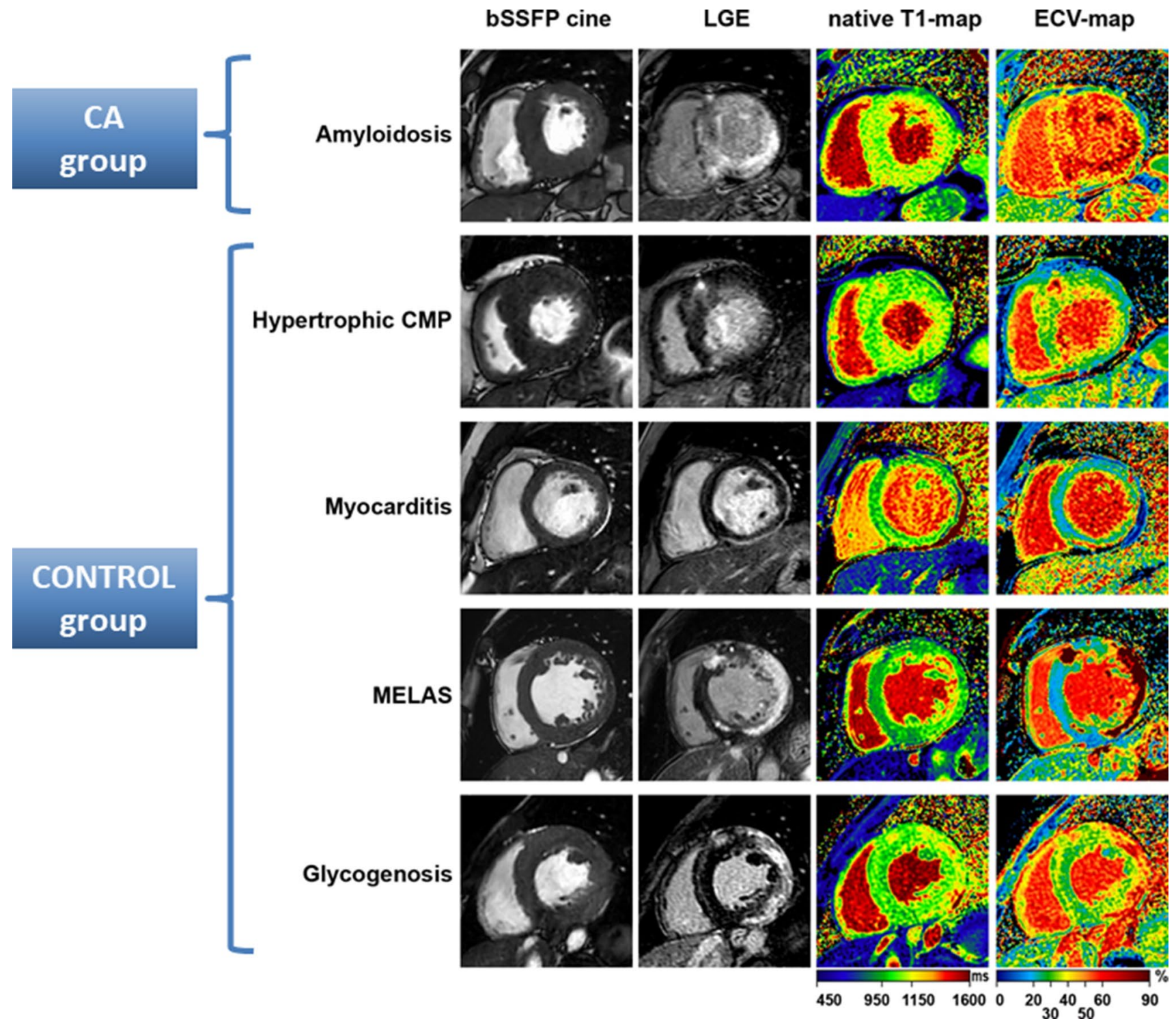

Fig. 1 Cardiovascular magnetic resonance (CMR) images of a patient with a characteristic pattern of late-gadolinium-enhancement (LGE) indicative of cardiac amyloidosis (CA) - in comparison to findings

\section{CMR acquisition}

CMR studies were performed on 1.5-T scanners (either Ingenia or Achieva, Philips Healthcare, Best, the Netherlands or Magnetom Aera, Siemens Medical Systems, Erlangen, Germany) in $N=151$ patients and on a 3.0-T scanner (Skyra, Siemens Medical Systems, Erlangen, Germany) in $N=9$ patients. The CMR protocol comprised cine- and LGE-imaging [magnitude only and additional phase-sensitive inversion recovery (PSIR) in case of need] as well as native and post-contrast T1-mapping using a modified Look Locker inversion recovery (MOLLI) sequence (performed in 3 out of 4 participating in the control group. Apart from LGE images, native T1-maps and ECV-maps are shown

centres and $N=93$ out of 160 patients)-allowing to measure extracellular volume fraction (ECV) of the myocardium. A CMR-based diagnosis of CA was made if a characteristic LGE-pattern indicative of CA was present. T1-mapping images were obtained in three short-axis views prior to as well as $15-20$ min after intravenous contrast agent administration (Gadobutrol or Gd-DOTA $0.15 \mathrm{mmol} / \mathrm{kg}$ ) to determine native T1- and ECV-values. Patients with chronic kidney disease (CKD) of stage 4 or 5 [i.e. glomerular filtration rate $(\mathrm{GFR}) \leq 30 \mathrm{ml} / \mathrm{min}$ ] were not excluded by default. Furthermore, patients with cardiac devices were only excluded if they showed fractured, epicardial or abandoned leads prior to the CMR study 
and/or poor image quality due to large device artefacts during the CMR scan.

\section{CMR data analysis}

CMR image analysis and interpretation were performed using commercially available software (cvi42-version 5.11.0, Circle Cardiovascular Imaging, Calgary, Alberta, Canada). Analysis of ventricular volumes and function as well as LV mass was made by contouring the endocardium and epicardium in short-axis cine-images. First, LGEimages were assessed visually and the pattern, distribution and extent of LGE was used to derive a CMR-based diagnosis of the underlying cardiac disease-as illustrated in detail elsewhere [18]. A CMR-based diagnosis of CA was supposed if a characteristic LGE-pattern indicative of CA (comprising all of the following criteria) was observed: (1) subendocardial to transmural LGE pattern predominantly in the basal LV segments, (2) no LGE distribution correlating to the perfusion area of a coronary artery and suggesting an ischemic myocardial scar, (3) no sharp demarcation and rather diffuse and extensive LGE pattern (Fig. 2a). In case of poor image quality of magnitude-only LGE images, additional PSIR-LGE were assessed (Fig. 2b). LGE was described as non-characteristic, when the aforementioned criteria were only partially fulfilled. In addition, the "Query Amyloid Late Enhancement" (QALE) score was reported as described in more detail elsewhere [13]. T1- and ECV-maps were assessed based on the consensus statement of SCMR. In those $N=9$ patients who were studied at 3.0-T, only ECV values (but not absolute T1-values) were considered for further analyses. All CMR data analyses were performed offline by experienced readers.

\section{Monoclonal protein studies}

Patients with suspected CA were tested for the presence of monoclonal gammopathy by studying the presence of monoclonal proteins in serum-suggestive of AL. First, sPE with high-resolution agarose gel allowed the demarcation of an $\mathrm{M}$-gradient. If an M-protein was present, sIFE and sFLC were performed to characterise its immunoglobulin chain type (heavy vs. light). The presence of a monoclonal protein was defined as an abnormal sFLC ratio (kappa-lambda ratio $<0.26$ or $>1.65$ ) or presence of a monoclonal band in sIFE.

\section{EMB procedure}

EMB specimens were obtained either from the RV septal wall (via a femoral vein access site using a 7F long-sheath with an angulated tip) and/or from the LV free wall (via a femoral artery access site using a 7F long-sheath without angulation following a retrograde approach) [14, 15]
(Supplemental Figure 1). Continuous ECG, blood pressure and pulse oximetry monitoring were performed throughout the whole procedure. Fluoroscopy was used for guidance of the long-sheath and bioptome, and for targeting the region of interest - which was defined in advance by non-invasive CMR. At least four EMB samples were collected from the RV and/or LV. Post-procedural echocardiography was performed to rule out or detect a pericardial effusion possibly caused by the biopsy procedure. Definition and assessment of biopsy complications was in accordance with previous studies [11].

\section{EMB workup (histology and immunohistochemistry)}

Histopathological and molecular pathological workup of biopsy samples were performed as described in detail previously $[11,16]$. Myocardial inflammation indicative of myocarditis was defined on the basis of immunohistochemical analyses based on hematoxylin/eosin and Masson trichrome stainings, respectively. Furthermore, Masson trichrome and hematoxylin/eosin staining as well as electron microscopy allowed EMB-based diagnoses of (amongst others) hypertrophic cardiomyopathy (HCM), dilated cardiomyopathy (DCM), cardiac sarcoidosis, transplant rejection, LV noncompaction cardiomyopathy (LVNC), mitochondrial cardiomyopathy, toxic cardiomyopathy, glycogen-storage disease and CA, respectively. Detection of amyloid was initially performed with Congo red staining of the formalin-fixed, paraffin-embedded myocardial tissue samples. Subsequently, immunohistochemistry allowed to identify the type of protein subunit with the use of monospecific antibodies reactive with the various types of amyloid. A negative result for CA was presumed only after thorough investigation by a specialised pathologist confirming negative histological and immunohistochemical examinations. Additional electron microscopy studies were performed depending on the preceding clinical and imaging suspicion and the pathologist's individual assessment.

\section{Genetic testing}

In those patients with EMB-proven ATTR amyloidosis, additional testing regarding the presence of a TTR gene mutation was in general aimed at. However, due to medicolegal reasons, genetic testing was only performed in $N=56$ out of 160 patients. Informed written consent for genetic testing in accordance with the Genetic Diagnostics Act (GenDG) was obtained. Thereafter, DNA extraction from the patient's blood with amplification by polymerase chain reaction assay and sequencing of the coding region of the TTR gene was performed. Mutations in the TTR gene associated with amyloid deposition are described elsewhere. 

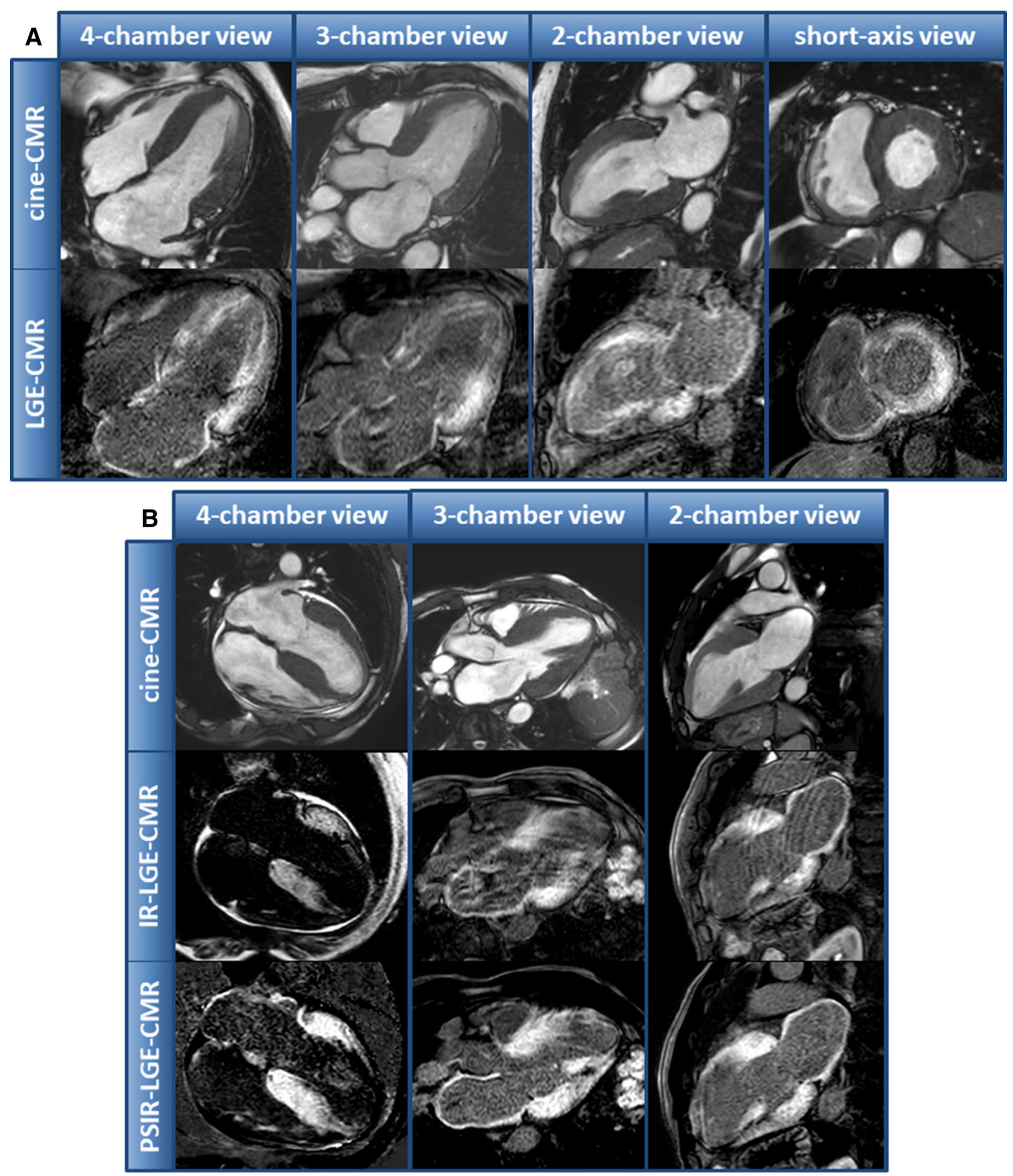

Fig. 2 a Example of a patient with ATTR amyloidosis and a characteristic late-gadolinium-enhancement (LGE)-pattern indicative of cardiac amyloidosis. b Example of another patient with ATTR amyloidosis and both magnitude only and additional phase-contrast inversion recovery (PSIR) LGE-images. PSIR-LGE-images show an improved image contrast and allow a better delineation of LGE in this example 


\section{Statistical analysis}

Statistical analysis was performed with SPSS (version 26.0, IBM Corp., Armonk, NY). Normal distribution of numeric variables was assessed with Shapiro-Wilk test. Continuous variables are expressed as mean \pm standard deviation whereas skewed variables as median \pm interquartile range. Categorical variables are expressed as frequency with percentage. An independent two-sample $T$ test was used for comparison of normally distributed data and Mann-Whitney $U$ test for non-normally distributed variables. The Fisher's exact test was used to compare non-continuous variables. Receiver operating characteristic curves (ROC) were analysed to assess the specificity and sensitivity of different CMR measurements to identify patients with CA within the whole study group. A $p$ value $<0.05$ was considered statistically significant. Sensitivity and specificity of CMR in diagnosing CA as well as positive (PPV) and negative predictive values (NPV) were calculated accordingly. Confidence intervals (CI) for sensitivity and specificity were calculated with the exact Clopper-Pearson method; CI for the predictive values was given as standard logit CI.

\section{Results}

\section{Study population}

The characteristics of the study population are summarised in Table 1. An EMB-based diagnosis of CA was made in $N=120$ patients (CA group) whereas $N=40$ patients demonstrated other diagnoses (CONTROL group). Males and females were equally distributed in the CA and CONTROL group $(p=0.48)$. Median age differed significantly between the two groups [75 (68-80) years in CA vs. 52 (46-61) years

Table 1 Patient characteristics

\begin{tabular}{lccc}
\hline & $\begin{array}{l}\text { CA group } \\
N=120\end{array}$ & $\begin{array}{l}\text { CONTROL group } \\
N=40\end{array}$ & $P$ value \\
\hline Male, $n(\%)$ & $100(83)$ & $31(78)$ & 0.48 \\
Age, years & $75(68-80)$ & $52(46-61)$ & $<\mathbf{0 . 0 0 1}$ \\
BMI, kg/m ${ }^{2}$ & $26( \pm 4)$ & $26( \pm 6)$ & 0.87 \\
Hypertension, $n(\%)$ & $87(73)$ & $22(55)$ & 0.05 \\
Diabetes, $n(\%)$ & $21(18)$ & $4(10)$ & 0.32 \\
High cholesterol, $n(\%)$ & $64(53)$ & $11(28)$ & $\mathbf{0 . 0 0 6}$ \\
Current smoker, $n(\%)$ & $15(13)$ & $15(38)$ & $\mathbf{0 . 0 0 1}$ \\
Coronary artery disease, & $48(40)$ & $8(20)$ & $\mathbf{0 . 0 2 3}$ \\
$n(\%)$ & & & $\mathbf{0 . 0 1 0}$ \\
Atrial fibrillation, $n(\%)$ & $59(49)$ & $10(25)$ & 0.07 \\
Chronic kidney disease & $11(9)$ & $0(0)$ & \\
$\quad($ GFR $<30$ ml/min), & & & \\
$n(\%)$ & & & \\
\hline
\end{tabular}

Bold $P$ values indicate $P<0.05$ in CONTROLs; $p<0.001]$ as expected due to the higher prevalence of CA in elderly patients. Atrial fibrillation was more common in CA patients ( 49 vs. $25 \%$; $p=0.010$ ).

\section{Endomyocardial biopsy (EMB) findings}

EMB was performed in all patients of the study group within a narrow time window after the CMR study [median time from CMR to $\mathrm{EMB}=1(0-3)$ months]. Regarding EMBassociated complications, there were four patients with myocardial perforation resulting in pericardial effusion with need for pericardiocentesis and one patient with a long-sheath induced non-sustained ventricular tachycardia. Importantly, all complications were treated quickly and successfully, and there were no detrimental or persistent complications at all. The overall complication rate of the EMB procedure was $3.13 \%$.

The diagnosis of CA in EMB samples was made according to the aforementioned histopathological definitions in $N=120$ patients. Amyloid-subtyping by targeted immunohistochemistry resulted in the diagnosis of cardiac ATTR amyloidosis in $N=92(77 \%)$ patients and cardiac AL amyloidosis in $N=28(23 \%)$ patients. In the remaining $N=40$ CONTROL patients without cardiac amyloidosis, however, with the presence of LV hypertrophy per definition, the following diagnoses were obtained: $N=10 \mathrm{HCM}, N=9$ myocarditis, $N=3$ cardiac sarcoidosis, $N=3$ transplant rejection, $N=3$ LVNC, $N=3$ DCM, $N=2$ mitochondrial cardiomyopathy, $N=2$ toxic cardiomyopathy, $N=1$ glycogen-storage disease, $N=1$ cardiac involvement in filaminopathy and $N=3$ non-ischemic cardiomyopathy of unknown aetiology.

\section{Genetic transthyretin testing results}

The majority of those $N=92$ patients with biopsy-proven ATTR amyloidosis underwent additional TTR gene testing for potential pathogenic TTR gene mutations. Mutations in the TTR gene indicating the presence of mATTR were detected in $N=6$ patients. The remaining patients with biopsy-proven ATTR (94\%) were classified as wtATTR.

\section{CMR findings}

The detailed anatomic, functional and structural CMR findings are listed in Table 2. Left ventricular ejection fraction (LV-EF) was significantly reduced in the CONTROL group compared to the CA group [40 (32-51) \% vs. 57 (46-62) $\% ; p<0.001]$. Accordingly, LV end diastolic volume (LVEDV) was markedly increased in the CONTROL group compared to the CA group $\left(116 \pm 41 \mathrm{ml} / \mathrm{m}^{2}\right.$ vs. $77 \pm 22 \mathrm{ml} / \mathrm{m}^{2}$; $p<0.001)$. Due to our study inclusion criteria, LV hypertrophy was present in all study patients-without a relevant difference in (global) LV mass between the CA and the 
Table 2 CMR parameters

\begin{tabular}{|c|c|c|c|}
\hline & $\begin{array}{l}\text { CA group } \\
N=120\end{array}$ & $\begin{array}{l}\text { CONTROL group } \\
N=40\end{array}$ & $P$ value \\
\hline \multicolumn{4}{|l|}{ Conventional CMR parameters } \\
\hline LV-EF, \% & $57(46-62)$ & $40(32-51)$ & $<0.001$ \\
\hline LV-EDV index, $\mathrm{ml} / \mathrm{m}^{2}$ & $77( \pm 22)$ & $116( \pm 41)$ & $<0.001$ \\
\hline LV-ESV index, $\mathrm{ml} / \mathrm{m}^{2}$ & $31(23-42)$ & $61(44-99)$ & $<0.001$ \\
\hline $\mathrm{LV}$ mass index, $\mathrm{g} / \mathrm{m}^{2}$ & $91(71-105)$ & $90(68-110)$ & 0.69 \\
\hline LV mass/volume ratio, $\mathrm{g} / \mathrm{ml}$ & $1.17(0.98-1.34)$ & $0.83(0.65-1.07)$ & $<0.001$ \\
\hline Max. LV wall thickness, mm & $19(17-22)$ & $14(13-18)$ & $<0.001$ \\
\hline RV-EF, \% & $49( \pm 11)$ & $51( \pm 10)$ & 0.41 \\
\hline $\mathrm{RV}$-EDV index, $\mathrm{ml} / \mathrm{m}^{2}$ & $82( \pm 22)$ & $92( \pm 28)$ & 0.06 \\
\hline RV-ESV index, $\mathrm{ml} / \mathrm{m}^{2}$ & $39(30-49)$ & $44(28-58)$ & 0.34 \\
\hline \multicolumn{4}{|l|}{ LGE } \\
\hline LGE extent, \% & $82(52-100)$ & $35(20-59)$ & $<0.001$ \\
\hline LGE QALE score, $n$ & $12(7-17)$ & $4(2-5)$ & $<0.001$ \\
\hline Characteristic LGE indicative of CA, $n(\%)$ & $114(95)$ & $1(2)$ & $<0.001$ \\
\hline \multicolumn{4}{|l|}{ T1-mapping } \\
\hline Native T1-mapping global, ms & $1152(1095-1245)$ & $1043(1036-1104)$ & $<0.001$ \\
\hline Native T1-mapping basal septal, ms & $1139(1086-1233)$ & $1062(1018-1137)$ & 0.001 \\
\hline \multicolumn{4}{|l|}{ ECV } \\
\hline ECV global, \% & $52( \pm 10)$ & $34( \pm 10)$ & $<0.001$ \\
\hline ECV basal septal, \% & $53(43-63)$ & $31(27-39)$ & $<0.001$ \\
\hline
\end{tabular}

Bold $P$ values indicate $P<0.05$

CONTROL group ( $p=0.69)$. However, a significantly higher maximal LV wall thickness was observed in the CA group compared to CONTROLs [19 [17-22] mm vs. 14 (13-18) $\mathrm{mm} ; p<0.001]$.

Regarding myocardial structure analyses, a non-ischemic, diffuse subendocardial to transmural pattern of LGE predominantly present in the LV basal to midventricular segments was detected in CA patients-with a substantially higher myocardial LGE extent in comparison to the CONTROL group [82 (52-100) \% vs. $35(20-59) \%, p<0.001]$. Accordingly, the assessment of the QALE score resulted in a significantly increased score in the CA group compared to the CONTROL group [12 (7-17) vs. $4(2-5) ; p<0.001]$. Furthermore, both native T1- and ECV-values were significantly increased in the CA group compared to the CONTROLs-not only in the basal septal wall but also in case of global LV assessment. In the CA group, there was no patient with elevated T1-/ECV-values without a characteristic pattern of LGE indicating CA (in those patients with available T1-maps). Noteworthy, neither native T1- nor ECV-values were available in those six patients with EMB-based diagnosis of CA, but absence of a LGE-pattern indicative of CA.

Subsequent ROC analyses showed excellent diagnostic yield for the parameters (a) predefined characteristic pattern of LGE indicative of CA, (b) global ECV—and a slightly reduced diagnostic value for (c) global native T1-mapping regarding the delineation of CA patients from the $\mathrm{CON}$ TROL group (Fig. 3). In particular, the parameter "characteristic pattern of LGE indicative of CA" (assessed as characteristic for CA according to the aforementioned criteria) showed an area under the curve (AUC) of 0.97 (95\%-CI: $0.89-1.00 ; p<0.001)$ whereas global ECV had an AUC of 0.87 (95\%-CI: $0.77-0.98 ; p<0.001)$ and global native $\mathrm{T} 1$ an AUC of 0.76 (95\%-CI: $0.60-0.92 ; p=0.003$ ).

LGE-imaging findings were further validated in comparison to biopsy findings. The respective LGE findings were classified dichotomously into "characteristic LGE pattern indicative of CA" vs. "LGE pattern NOT unequivocally indicative of CA". There was only one patient with a "characteristic LGE pattern indicative of CA" that could not be verified by EMB. Review of this patient's case showed that a RV biopsy was performed and that the bioptome was not optimally positioned in the basal to mid part of the interventricular septum but rather in the apical part. Hence, a potential "sampling error" due to suboptimal positioning of the bioptome may have resulted in a "false-negative" EMB result. Furthermore, there were 6 out of 120 (5\%) patients with EMB-proven CA who did not show a "characteristic LGE pattern indicative of CA" upon CMR. In comparison to the gold standard EMB, the non-invasive CMR parameter "characteristic LGE pattern indicative of CA" showed a sensitivity of $95 \%$ and a specificity of $98 \%$ for the diagnosis 


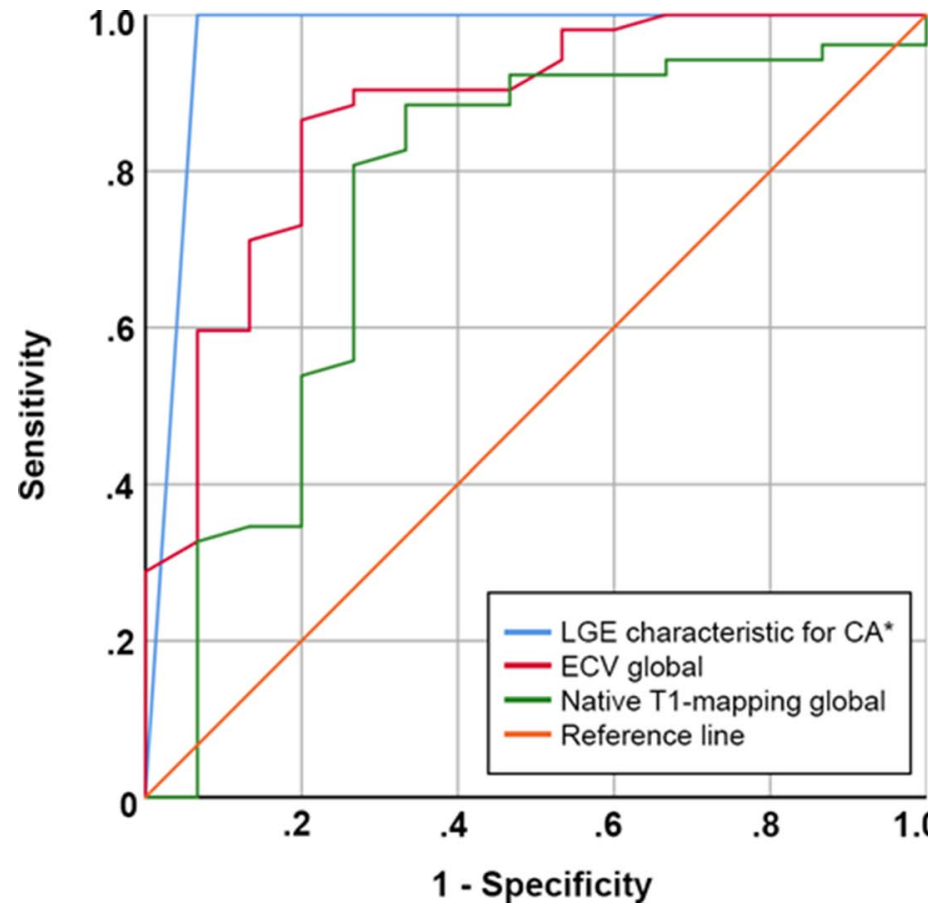

\begin{tabular}{cccc}
\hline CMR parameter & AUC & $\mathbf{9 5 \% - C l}$ & p-value \\
\hline $\begin{array}{c}\text { LGE characteristic } \\
\text { for CA* }\end{array}$ & 0.97 & $0.89-1.00$ & $<0.001$ \\
$\begin{array}{c}\text { ECV global } \\
\begin{array}{c}\text { Native T1-mapping } \\
\text { global }\end{array}\end{array}$ & 0.87 & $0.77-0.98$ & $<0.001$ \\
\hline
\end{tabular}

Fig. 3 Receiver operating characteristic (ROC) curves illustrating the diagnostic yield of different CMR parameters regarding the diagnosis of cardiac amyloidosis (CA)

Table 3 Sensitivity and specificity of CMR for the diagnosis of CA compared to EMB

\begin{tabular}{llll}
\hline & $\begin{array}{l}\text { Characteristic } \\
\text { LGE indicative } \\
\text { of CA }\end{array}$ & $\begin{array}{l}\text { LGE pattern } \\
\text { NOT indicative } \\
\text { of CA }\end{array}$ & $\begin{array}{l}\text { Sensitivity and } \\
\text { specificity (CI), \% }\end{array}$ \\
\hline $\begin{array}{l}\text { EMB- } \\
\text { proven } \\
\text { presence } \\
\text { of CA, }\end{array}$ & $114(95)$ & $6(5)$ & $\begin{array}{c}95(89-98) \text { sensi- } \\
\text { tive }\end{array}$ \\
$\begin{array}{l}N=120 \\
\text { EMB nega- } \\
\text { tive for } \\
\text { CA, } N=40\end{array}$ & $1(2)$ & $39(98)$ & \\
\hline
\end{tabular}

of CA (Table 3). Accordingly, the positive predictive value (PPV) of LGE-CMR for the diagnosis of CA was $99 \%$ (95\%CI: $94-100 \%)$ whereas the respective negative predictive value (NPV) was $87 \%$ (95\%-CI: 75-93\%).

\section{Combination of CMR and monoclonal protein findings}

Presence of monoclonal proteins in the serum suggestive of AL was found in all $N=28$ patients with biopsy-proven AL amyloidosis. In contrast, there were 6 out of $92(7 \%)$ patients with biopsy-proven ATTR amyloidosis who also showed presence of serum monoclonal proteins - in the absence of AL amyloidosis. Hence, monoclonal protein findings in these six patients with a median age of 73 years were assessed as monoclonal gammopathy of undetermined significance (MGUS). The combined finding of a "characteristic LGE pattern indicative of CA" (upon CMR) AND negative monoclonal protein studies was $98 \%$ specific for the diagnosis of cardiac ATTR amyloidosis. Moreover, such a combined finding showed a PPV of $99 \%$

Table 4 Combination of CMR results with monoclonal protein studies (MPS)

\begin{tabular}{llll}
$\begin{array}{l}\text { Characteristic LGE indicative of } \\
\text { CA + negative MPS }\end{array}$ & $\begin{array}{l}\text { LGE pattern NOT indicative of } \\
\text { CA and/or positive MPS }\end{array}$ & $\begin{array}{l}\text { Sensitivity and specificity } \\
\text { (CI), \% }\end{array}$ & PPV and NPV (CI), \% \\
\hline 83 & 9 & $90(82-95)$ sensitive & NPV $=88(80-93)$ \\
1 & 67 & $99(92-100)$ specific & PPV $=99(92-100)$
\end{tabular}


(95\%-CI 92-100\%) for the diagnosis of cardiac ATTR amyloidosis when compared to biopsy findings (Table 4).

\section{Discussion}

To the best of our knowledge, this is the largest multicentre German study that evaluated the diagnostic value of CMR in comparison to EMB-proven CA. The results of our present study are based on real-world clinical data from four German centres that are highly experienced in both (a) conducting CMR studies (with a high volume of $>2.000$ CMR studies per year and centre) and (b) performing EMBs (with a high volume of $>100$ EMB procedures per year and centre) for workup of suspected cardiomyopathies of unknown origin. This real-world German experience clearly illustrates that (a) non-invasive CMR allows to diagnose the presence of CA with a high sensitivity of 95\% and an even higher specificity of $98 \%$ and that (b) the combined finding of a positive CMR study (indicative of $\mathrm{CA}$ ) and negative monoclonal protein studies is not only highly specific for the diagnosis of cardiac ATTR amyloidosis (specificity of $98 \%$ ) but also highly trustable with a PPV of $99 \%$ (for the diagnosis of cardiac ATTR amyloidosis)—proven by biopsy results.

Already in 2008, the Stuttgart group assessed the diagnostic value of LGE-CMR for the diagnosis of CA in a rather small study group of 33 patients [6]: cardiac amyloidosis was detected by EMB in 15 out of 33 patients who were studied more than a decade ago (on 1.5-T Magnetom Sonata at that time). Using the characteristic LGEpattern indicative of $\mathrm{CA}$ as a diagnostic criterion, they obtained a sensitivity of $80 \%$ and a specificity of $94 \%$ for the diagnosis of CA-at that time. Obviously, both clinical experience in assessing LGE-CMR images as well as CMR-techniques have tremendously improved since that time allowing (amongst others) more experienced and more accurate assessment of LGE-images today [17-19]. Hence, the results of the present multi-centre study nicely illustrate this overall progress and clearly demonstrate that both sensitivity and specificity of LGE-based diagnosis of CA have improved.

Obviously, today cardiac workup of patients with suspected CA should be based on multi-parametric CMR including T1-mapping and ECV measurement-and not limited to LGE-CMR - since several studies have shown a superior diagnostic value of mapping-based approaches compared to conventional LGE-imaging $[7,8]$. In principle, native T1-mapping and ECV measurement are highly suitable tools to detect (and quantify) even subtle amyloid deposits in the extracellular space of the myocardium [5, 20]. However, there are still some puzzling data regarding the comparison of native T1- and ECV-values in case of CA, e.g. the "native T1 versus ECV paradox" in CA that was recently addressed in detail elsewhere [21]. In a recent meta-analysis, a total of 18 diagnostic $(N=2015)$ and 13 prognostic CMR studies $(N=1483)$ using native T1, ECV or LGE to diagnose and prognosticate CA were included for analysis [22]. According to this meta-analysis, the parameter ECV showed a significantly higher diagnostic odds ratio for CA than conventional LGEassessment. However, there was no significant difference between LGE-assessment and native T1 for sensitivity, specificity and diagnostic odds ratio-regarding the diagnosis of CA. Our present study does not allow to safely compare the diagnostic value of T1-mapping and/or ECV measurement in comparison to LGE-imaging since mapping was not performed in all patients. In this context, it needs to be considered that cut-off values for native T1 or ECV derived from ROC analyses (in a specific group of study patients) for ruling in or out the presence of CA are-amongst others-determined by native T1- and ECV-values of the respective control group. A different composition of the control group (e.g. higher percentage of HCM patients with extensive myocardial fibrosis vs. lower percentage of healthy controls without fibrosis) will result in different cut-off values. Hence, comparisons of diagnostic CMR parameters based on ROC analyses (using cut-off values) need to be considered carefully-with a special attention to the control group of the underlying study - and single-centre results cannot be transferred to other centres by default.

In the last years, innumerable studies addressing noninvasive diagnosis of CA were published by colleagues from the National Amyloidosis Centre in London/UKdeserving the designation the London/UK experience [7, $12,17,23]$. Their study results moved the diagnostic field forward and substantially contributed to current recommendations regarding the diagnostic approach in suspected CA $[12,24,25]$. Due to the efforts of these colleagues, bone scintigraphy was established as a substantial non-invasive method to diagnose CA [24-27]. However, this London/ UK experience needs to be considered with some caution and a non-reflected transfer of the UK-based results to other EU countries should be avoided. As outlined by these colleagues themselves, the patients presenting to this central UK centre are not "unselected", but rather referred to this centre of experience with suspected CA. Consequently, the cohort of patients studied at this centre-and in particular, the "control group" used in their studies-does not reflect an "unselected" cardiology population of patients. Obviously, the same is true for our present study due to our methodological approach in selecting patients. Importantly, the patients of this study were initially referred as "outpatients" to our specialised cardiomyopathy centres (mostly by resident cardiologists due to suspected cardiomyopathy for further 
diagnostic workup). Hence, we neither included, e.g. decompensated inpatients presenting directly to our emergency or intensive care units who also underwent CMR and/or biopsy workup during their further hospitalisation, nor did we study an unselected cardiology population. However, our control group comprising only patients with LV hypertrophy should be more appropriate regarding validation of imaging methods for the diagnosis of CA.

To appropriately assess the value of the present CMR results in comparison to previous bone scintigraphy results (e.g. the London/UK experience), a careful look on the data of Gillmore et al. is required [12]: in a first step, Gillmore et al. analysed the data of 374 patients with EMB and defined a "positive" bone scintigraphy indicative of CA as cardiac tracer uptake of either grade 1,2 or 3 (vs. grade 0 as "normal" finding). This approach resulted in a sensitivity of $88 \%$ and a specificity of $87 \%$ for bone scintigraphy to detect CA (independent of subtype). In a second step, the authors focused on those patients with ATTR-CA and received a very high sensitivity of $>99 \%$ for bone scintigraphy to detect ATTR-CAbut a rather low specificity of $68 \%$. In a third step, the authors changed their "positive" definition of bone scintigraphy and presumed that a cardiac tracer uptake of only grade 2 or 3 was indicative of ATTR-CA (vs. grade 0 or 1 defined as "normal/negative" findings). Such a different assumption resulted in a re-grouping of 42 (out of 374 patients $=11 \%$ ) from the positive group to the negative group, and in a sensitivity of $91 \%$ and a specificity of $87 \%$ for bone scintigraphy to detect ATTR-CA. In comparison, in our present CMR study, the presence of CA was diagnosed based on LGE-CMR with a high sensitivity of $95 \%$ and an even higher specificity of $98 \%$-without re-grouping of study patients by changing assumptions.

Furthermore, Gillmore et al. analysed the specificity and PPV of a "positive" bone scintigraphy indicative of CA (defined as cardiac tracer uptake of either grade 2 or 3 vs. grade 0 or 1 as "normal/negative") for ATTRCA when combined with the absence of monoclonal proteins in $N=374$ patients with EMB [12]: they obtained a specificity as well as PPV of $100 \%$ for the diagnosis of ATTR-CA. Based on this finding, they established their approach and diagnostic algorithm of bone scintigraphybased non-biopsy diagnosis of ATTR-CA that entered some recommendation papers [24-26] - and is questioned by some interesting reports $[28,29]$. In contrast, in our present study, the combined finding of a "positive" CMR study indicative of CA and "negative" monoclonal protein studies resulted in a similar specificity of $98 \%$ and PPV of 99\% for the diagnosis of ATTR-CA (compared to biopsy findings). Hence, our present results clearly suggest that non-invasive CMR allows both (a) to safely diagnose the presence of CA and (b) to further verify the presence of ATTR-CA by additional negative monoclonal protein studies-similar to bone scintigraphy. Obviously, in case of such clear CMR findings there is no need for additional diagnostic methods (such as bone scintigraphy) — and the diagnostic algorithm suggested in some recommendation papers needs to be carefully revised. Based on the present German experience, we suggest the diagnostic algorithm illustrated in Fig. 4.

Finally, one may argue that additional EMBs are not required in those patients with the combined finding of a "positive" CMR study indicative of CA and "negative" monoclonal protein studies-similar to the scintigraphic approach of Gillmore et al. [12]. However, since our knowledge on the underlying pathophysiology of amyloid deposition in the human myocardium is still very limited, and since CA is not only characterised by extracellular accumulation of amyloid fibrils but also by myocardial oedema, inflammation, and myocyte hypertrophy (with variable degrees of each pathophysiology potentially resulting in differing effects on native T1, ECV and LGE-pattern), we still need the histopathological information from EMBs in order to better understand our non-invasive imaging findings. This issue will be even far more important considering upcoming specific therapies to treat ATTR-CA: we will need the EMB information in order to better understand both (a) the therapeutic effect of the respective medication/ therapy and (b) the change in non-invasive cardiac imaging parameters that will be used for disease monitoring. Hence, considering the low risk of EMB complication even in patients with $\mathrm{CA}$ - as confirmed in the present study, we suggest to continue to biopsy even patients with the combined finding of a "positive" CMR study indicative of CA and "negative" monoclonal protein studies. For the remaining patients, there is no doubt that EMB still represents the gold standard for workup of non-ischemic, unexplained cardiomyopathy $[9,10,16]$.

Obviously, the EMB complication rate of $3.13 \%$ in the present study is somewhat higher compared to previous studies suggesting a major complication rate of $0.64 \%$ for LV-EMB and of $0.82 \%$ for RV-EMB - proving that EMB is a safe procedure. Moreover, there are reports that mentioned a myocardial perforation risk (= major complication) in CA patients in up to $17.1 \%$ in case of RVEMB and up to $6.6 \%$ in case of LV-EMB (Kristen et al. Am J Hematology 2007;82:327-333)—and suggested a more "fragile" myocardium in case of CA with a higher risk of myocardial perforation in particular in case of RV-EMB. Obviously, the complication rate of EMB in the present study was tremendously lower as reported in the aforementioned study of Kristen et al. In the present study, myocardial perforation occurred in four patients all belonging to the CA group-supporting the notion of a "fragile" myocardium in case of advanced CA. However, 


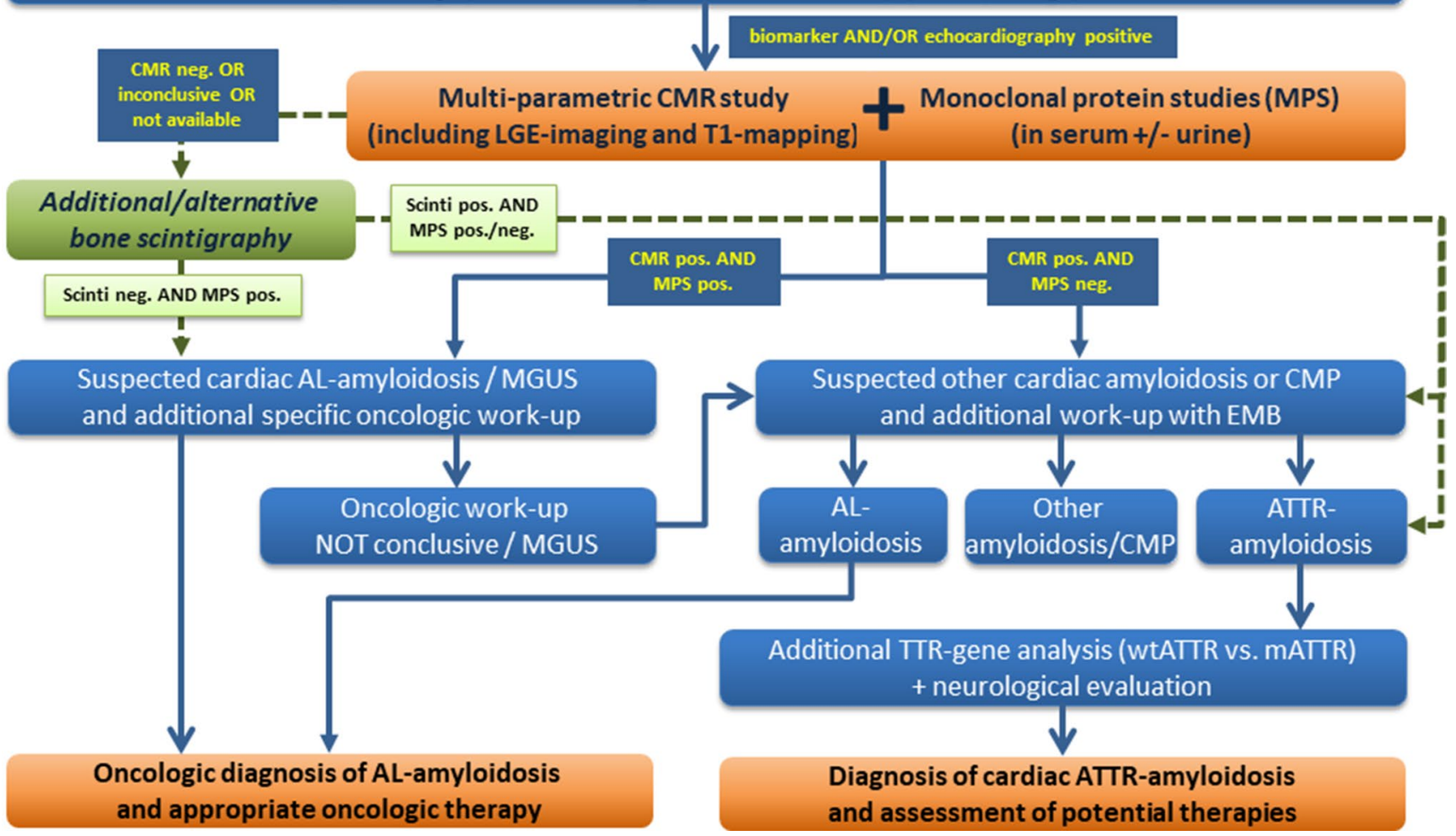

Fig. 4 Schematic diagram representing the suggested diagnostic pathway for workup of cardiac amyloidosis (CA)

these complications occurred when a "stiff" bioptome was used whereas no complications were observed when using more smooth/elastic bioptomes. In our opinion, the risk of perforation in case of EMB is driven by (a) the properties of the bioptome, (b) the experience of the interventionalist and (c) the composition of the myocardium - with a potentially more "fragile" myocardium in case of CA. Therefore, EMB procedures in patients with suspected CA are quite safe when they are performed by experienced interventionalists and preferably taken from the LV using smooth bioptomes.

Finally, the issue of balancing diagnostic yield vs. procedure-associated risk and costs is highly important regarding clinical decision-making in daily routine. However, a discussion on this issue would go far beyond the scope of the present work and should be performed by expert panels based on more comprehensive data regarding all of these issues. Nevertheless, we believe that the "diagnostic" data presented in our manuscript will help such expert panels aiming at balancing diagnostic yield, procedure safety, method availability, costs and practical consequences.

\section{Limitations}

Similar to other previous studies, our study group does not reflect an "unselected" cardiology population of patients and comprised only those suffering from symptoms of heart failure in the presence of left ventricular (LV) hypertrophy that could not be explained by abnormal loading conditions. Obviously, we cannot exclude a potential selection bias by selected patient referral to our specialised cardiomyopathy centres. However, we believe that our approach is appropriate for evaluating the diagnostic yield of CMR for the workup of CA (based on real-world clinical data), since our CONTROL group did not comprise healthy patients or patients without relevant structural abnormalities - but rather severely diseased patients with other cardiac diseases presenting to four German specialised cardiomyopathy centres. Moreover, T1-mapping and ECV data were not available in all patients-limiting the value of comparative analyses of LGE vs. native T1 and/ or ECV. However, LGE-pattern per se showed a convincing diagnostic yield and T1-mapping-based approaches 
will obviously "strengthen"- -but not "worsen"—-this yield and particularly allow a monitoring of myocardial amyloid load over time.

\section{Conclusion}

Non-invasive CMR shows an excellent diagnostic accuracy and yield regarding CA. When combined with monoclonal protein studies, CMR can differentiate ATTR from AL with high accuracy and predictive value. However, invasive EMB remains a safe invasive gold standard and allows to differentiate CA from other cardiomyopathies that can also cause LV hypertrophy.

Author contributions GC and MB participated in the CMR exams, carried out the data and statistical analysis, and wrote some paragraphs of the draft version of the manuscript. AE, GT, JV and ZS participated in the CMR exams and in the analysis of the CMR data. CS, BP, HR, $\mathrm{HM}$, and SK participated in data collection and analyses, and critically reviewed the manuscript. AY supervised the study, wrote major paragraphs of the manuscript, critically reviewed the final manuscript and drafted the manuscript. All authors read and approved the final manuscript.

Funding Open Access funding enabled and organized by Projekt DEAL.

Availability of data and materials The datasets used and/or analysed during the current study are available from the corresponding author on reasonable request.

\section{Compliance with ethical standards}

\section{Conflict of interest None.}

Ethics approval and consent to participate The study protocol complies with the Declaration of Helsinki. Ethics approval was obtained from local authorities and written informed consent was obtained from the participants.

\section{Consent for publication N/A.}

Open Access This article is licensed under a Creative Commons Attribution 4.0 International License, which permits use, sharing, adaptation, distribution and reproduction in any medium or format, as long as you give appropriate credit to the original author(s) and the source, provide a link to the Creative Commons licence, and indicate if changes were made. The images or other third party material in this article are included in the article's Creative Commons licence, unless indicated otherwise in a credit line to the material. If material is not included in the article's Creative Commons licence and your intended use is not permitted by statutory regulation or exceeds the permitted use, you will need to obtain permission directly from the copyright holder. To view a copy of this licence, visit http://creativecommons.org/licenses/by/4.0/.

\section{References}

1. Connors LH, Sam F, Skinner M, Salinaro F, Sun F, Ruberg FL, Berk JL, Seldin DC (2016) Heart failure resulting from age-related cardiac amyloid disease associated with wild-type transthyretin: a prospective. Observ Cohort Study Circ 133(3):282-290

2. Merlini G, Bellotti V (2003) Molecular mechanisms of amyloidosis. N Engl J Med 349(6):583-596

3. Wechalekar AD, Gillmore JD, Hawkins PN (2016) Systemic amyloidosis. Lancet 387(10038):2641-2654

4. Gillmore JD, Damy T, Fontana M, Hutchinson M, Lachmann HJ, Martinez-Naharro A, Quarta CC, Rezk T, Whelan CJ, GonzalezLopez E, Lane T, Gilbertson JA, Rowczenio D, Petrie A, Hawkins PN (2018) A new staging system for cardiac transthyretin amyloidosis. Eur Heart J 39(30):2799-2806

5. Messroghli DR, Moon JC, Ferreira VM, Grosse-Wortmann L, He T, Kellman P, Mascherbauer J, Nezafat R, Salerno M, Schelbert EB, Taylor AJ, Thompson R, Ugander M, van Heeswijk RB, Friedrich MG (2017) Clinical recommendations for cardiovascular magnetic resonance mapping of $\mathrm{T} 1, \mathrm{~T} 2, \mathrm{~T} 2 *$ and extracellular volume: a consensus statement by the Society for Cardiovascular Magnetic Resonance (SCMR) endorsed by the European Association for Cardiovascular Imaging (EACVI). J Cardiovasc Magn Reson 19(1):75

6. Vogelsberg H, Mahrholdt H, Deluigi CC, Yilmaz A, Kispert EM, Greulich S, Klingel K, Kandolf R, Sechtem U (2008) Cardiovascular magnetic resonance in clinically suspected cardiac amyloidosis: noninvasive imaging compared to endomyocardial biopsy. J Am Coll Cardiol 51(10):1022-1030

7. Fontana M, Banypersad SM, Treibel TA, Maestrini V, Sado DM, White SK, Pica S, Castelletti S, Piechnik SK, Robson MD, Gilbertson JA, Rowczenio D, Hutt DF, Lachmann HJ, Wechalekar AD, Whelan CJ, Gillmore JD, Hawkins PN, Moon JC (2014) Native T1 mapping in transthyretin amyloidosis. JACC Cardiovasc Imaging 7(2):157-165

8. Martinez-Naharro A, Kotecha T, Norrington K, Boldrini M, Rezk T, Quarta C, Treibel TA, Whelan CJ, Knight DS, Kellman P, Ruberg FL, Gillmore JD, Moon JC, Hawkins PN, Fontana M (2019) Native T1 and extracellular volume in transthyretin amyloidosis. JACC Cardiovasc Imaging 12(5):810-819. https://doi. org/10.1016/j.jcmg.2018.02.006

9. Ardehali H, Qasim A, Cappola T, Howard D, Hruban R, Hare JM, Baughman KL, Kasper EK (2004) Endomyocardial biopsy plays a role in diagnosing patients with unexplained cardiomyopathy. Am Heart J 147(5):919-923

10. Cooper LT, Baughman KL, Feldman AM, Frustaci A, Jessup M, Kuhl U, Levine GN, Narula J, Starling RC, Towbin J, Virmani R (2007) The role of endomyocardial biopsy in the management of cardiovascular disease: a scientific statement from the American Heart Association, the American College of Cardiology, and the European Society of Cardiology Endorsed by the Heart Failure Society of America and the Heart Failure Association of the European Society of Cardiology. Eur Heart J 28(24):3076-3093

11. Yilmaz A, Kindermann I, Kindermann M, Mahfoud F, Ukena C, Athanasiadis A, Hill S, Mahrholdt H, Voehringer M, Schieber M, Klingel K, Kandolf R, Bohm M, Sechtem U (2010) Comparative evaluation of left and right ventricular endomyocardial biopsy: differences in complication rate and diagnostic performance. Circulation 122(9):900-909

12. Gillmore JD, Maurer MS, Falk RH, Merlini G, Damy T, Dispenzieri A, Wechalekar AD, Berk JL, Quarta CC, Grogan M, 
Lachmann HJ, Bokhari S, Castano A, Dorbala S, Johnson GB, Glaudemans AW, Rezk T, Fontana M, Palladini G, Milani P, Guidalotti PL, Flatman K, Lane T, Vonberg FW, Whelan CJ, Moon JC, Ruberg FL, Miller EJ, Hutt DF, Hazenberg BP, Rapezzi C, Hawkins PN (2016) Nonbiopsy diagnosis of cardiac transthyretin amyloidosis. Circulation 133(24):2404-2412

13. Dungu JN, Valencia O, Pinney JH, Gibbs SD, Rowczenio D, Gilbertson JA, Lachmann HJ, Wechalekar A, Gillmore JD, Whelan CJ, Hawkins PN, Anderson LJ (2014) CMR-based differentiation of AL and ATTR cardiac amyloidosis. JACC Cardiovasc Imaging 7(2):133-142

14. Tschope C, Kherad B, Schultheiss HP (2015) How to perform an endomyocardial biopsy? Turk Kardiyol Dern Ars 43(6):572-575

15. Yilmaz A, Klingel K, Kandolf R, Sechtem U (2012) Endomyocardial biopsy. The PCR-EAPCI textbook, 1st edn, Europa Edition. http://www.pcronline.com/eurointervention/textbook/pcr-textb ook/

16. Baccouche H, Mahrholdt H, Meinhardt G, Merher R, Voehringer M, Hill S, Klingel K, Kandolf R, Sechtem U, Yilmaz A (2009) Diagnostic synergy of non-invasive cardiovascular magnetic resonance and invasive endomyocardial biopsy in troponinpositive patients without coronary artery disease. Eur Heart J 30(23):2869-2879

17. Fontana M, Pica S, Reant P, Abdel-Gadir A, Treibel TA, Banypersad SM, Maestrini V, Barcella W, Rosmini S, Bulluck H, Sayed RH, Patel K, Mamhood S, Bucciarelli-Ducci C, Whelan CJ, Herrey AS, Lachmann HJ, Wechalekar AD, Manisty CH, Schelbert EB, Kellman P, Gillmore JD, Hawkins PN, Moon JC (2015) Prognostic value of late gadolinium enhancement cardiovascular magnetic resonance in cardiac amyloidosis. Circulation 132(16):1570-1579

18. Francis R, Kellman P, Kotecha T, Baggiano A, Norrington K, Martinez-Naharro A, Nordin S, Knight DS, Rakhit RD, Lockie T, Hawkins PN, Moon JC, Hausenloy DJ, Xue H, Hansen MS, Fontana M (2017) Prospective comparison of novel dark blood late gadolinium enhancement with conventional bright blood imaging for the detection of scar. J Cardiovasc Magn Reson 19(1):91

19. Kellman P, Xue H, Olivieri LJ, Cross RR, Grant EK, Fontana M, Ugander M, Moon JC, Hansen MS (2016) Dark blood late enhancement imaging. J Cardiovasc Magn Reson 18(1):77

20. Bietenbeck M, Florian A, Shomanova Z, Klingel K, Yilmaz A (2017) Novel CMR techniques enable detection of even mild autoimmune myocarditis in a patient with systemic lupus erythematosus. Clin Res Cardiol 106(7):560-563

21. Yilmaz A (2018) The "native T1 versus extracellular volume fraction paradox" in cardiac amyloidosis: answer to the million-dollar question? JACC Cardiovasc Imaging

22. Pan JA, Kerwin MJ, Salerno M (2020) Native T1 mapping, extracellular volume mapping, and late gadolinium enhancement in cardiac amyloidosis: a meta-analysis. JACC Cardiovasc Imaging 13(6):1299-1310

23. Baggiano A, Boldrini M, Martinez-Naharro A, Kotecha T, Petrie A, Rezk T, Gritti M, Quarta C, Knight DS, Wechalekar AD, Lachmann HJ, Perlini S, Pontone G, Moon JC, Kellman P, Gillmore JD, Hawkins PN, Fontana M (2020) Noncontrast magnetic resonance for the diagnosis of cardiac amyloidosis. JACC Cardiovasc Imaging 13(1 Pt 1):69-80

24. Dorbala S, Vangala D, Semer J, Strader C, Bruyere JR Jr, Di Carli MF, Moore SC, Falk RH (2014) Imaging cardiac amyloidosis: a pilot study using (1)(8)F-florbetapir positron emission tomography. Eur J Nucl Med Mol Imaging 41(9):1652-1662

25. Dorbala S, Vangala D, Bruyere J Jr, Quarta C, Kruger J, Padera R, Foster C, Hanley M, Di Carli MF, Falk R (2014) Coronary microvascular dysfunction is related to abnormalities in myocardial structure and function in cardiac amyloidosis. JACC Heart Fail 2(4):358-367

26. Maurer MS, Bokhari S, Damy T, Dorbala S, Drachman BM, Fontana M, Grogan M, Kristen AV, Lousada I, Nativi-Nicolau J, Cristina QC, Rapezzi C, Ruberg FL, Witteles R, Merlini G (2019) Expert consensus recommendations for the suspicion and diagnosis of transthyretin cardiac amyloidosis. Circ Heart Fail 12(9): $\mathrm{e} 006075$

27. Yilmaz A, Bauersachs J, Kindermann I, Klingel K, Knebel F, Meder B, Morbach C, Nagel E, Schulze-Bahr E, Aus Dem Siepen F, Frey N (2019) Position statement of the German Society of Cardiology (DGK) regarding "diagnosis and therapy of cardiac amyloidosis." Der Kardiologe 3:264-291

28. Layoun ME, Desmarais J, Heitner SB, Masri A (2020) Hot hearts on bone scintigraphy are not all amyloidosis: hydroxychloroquineinduced restrictive cardiomyopathy. Eur Heart J 41(25):2414

29. Pilebro B, Suhr OB, Naslund U, Westermark P, Lindqvist P, Sundstrom T (2016) (99m)Tc-DPD uptake reflects amyloid fibril composition in hereditary transthyretin amyloidosis. Ups J Med Sci 121(1):17-24

\section{Affiliations}

\section{Grigorios Chatzantonis $^{1} \cdot$ Michael Bietenbeck $^{1} \cdot$ Ahmed Elsanhoury $^{2} \cdot$ Carsten Tschöpe $^{2,3} \cdot$ Burkert Pieske $^{4}$. Gloria Tauscher $^{5}$. Julia Vietheer ${ }^{6,7} \cdot$ Zornitsa Shomanova $^{1} \cdot$ Heiko Mahrholdt ${ }^{5}$. Andreas Rolf ${ }^{6,7} \cdot$ Sebastian Kelle ${ }^{4}$. Ali Yilmaz ${ }^{1}[$}

Grigorios Chatzantonis

grigorios.chatzantonis@ukmuenster.de

Michael Bietenbeck

michael.bietenbeck@ukmuenster.de

Ahmed Elsanhoury

ahmed.elsanhoury@charite.de

Carsten Tschöpe

carsten.tschoepe@charite.de
Burkert Pieske

pieske@dhzb.de

Gloria Tauscher

gloria.tauscher@rbk.de

Julia Vietheer

j.vietheer@kerckhoff-klinik.de

Zornitsa Shomanova

zornitsa.shomanova@ukmuenster.de 
Heiko Mahrholdt

heiko.mahrholdt@rbk.de

Andreas Rolf

a.rolf@kerckhoff-klinik.de

Sebastian Kelle

kelle@dhzb.de

1 Department of Cardiology I, University Hospital Münster, Albert-Schweitzer-Campus 1, Building A1, 48149 Münster, Germany

2 Department of Cardiology, Centre for Regenerative Therapies (BCRT), Campus Virchow and Berlin Institute of Health (BIH), Berlin, Charite, Berlin, Germany
DZHK (German Centre for Cardiovascular Research), partner site Berlin, Charite, Berlin, Germany

4 Department of Internal Medicine/Cardiology, Deutsches Herzzentrum Berlin, Berlin, Germany

5 Department of Cardiology, Robert-Bosch-Medical Centre, Stuttgart, Germany

6 Department of Cardiology, Kerckhoff Hospital, University Giessen, Bad Nauheim, Germany

7 DZHK (German Centre for Cardiovascular Research), partner site Rhine Main, Frankfurt, Germany 